\title{
FRACIONAMENTO E CINÉTICA DA FERMENTAÇÃO RUMINAL in vitro DOS CARBOIDRATOS DE CINCO VARIEDADES DE CANA-DE-AÇÚCAR
}

\author{
Pedro Gomes da Cruz, ${ }^{1}$ Mauro Pereira de Figueiredo, ${ }^{2}$ Luiz Gustavo Ribeiro Pereira, ${ }^{3}$ \\ Keityane Boone Bergamaschi, ${ }^{4}$ Carlindo Santos Rodrigues ${ }^{5}$ e Carmen Lúcia de Souza Rech ${ }^{6}$ \\ 1. Doutorando em Ciência Animal e Pastagens pela Escola Superior de Agricultura "Luiz de Queiroz". \\ E-mail:pgcruz@esalq.usp \\ 2. Professor titular da Universidade Estadual do Sudoeste da Bahia \\ 3. Pesquisador da Embrapa Gado de Leite \\ 4. Especialista em Alimentação e Nutrição de Cães e Gatos pela Universidade Federal de Lavras \\ 5. Doutorando em zootecnia na Universidade Federal de Viçosa \\ 6. Professor assistente da Universidade Estadual do Sudoeste da Bahia.
}

\section{RESUMO}

O objetivo deste trabalho foi determinar a composição química, o fracionamento dos carboidratos e a cinética da fermentação ruminal in vitro dos carboidratos não fibrosos e carboidratos fibrosos de cinco variedades de cana-de-açúcar. A variedade SP79-1011 apresentou o maior teor de nutrientes digestíveis totais (65,9\%), seguida das variedades Java, RB72454, SP80-1842 e $\mathrm{RB} 765418$. A produção acumulada de gases na matéria seca às 48 e 96 horas de incubação in vitro foi maior para a variedade SP791011, apresentando diferença significativa em comparação com as variedades RB765418, RB72454 e SP80-1842, exceção feita à variedade Java, que apresentou valores semelhantes à primeira. $\mathrm{O}$ fator de partição da matéria seca diminuiu com o aumento no tempo de incubação, sendo que não houve diferença entre as variedades avaliadas. As características do ciclo de produção influenciaram na fermentação ruminal in vitro nas variedades avaliadas, sendo que as de ciclo precoce SP80-1842 e RB765418 foram as que apresentaram resultados inferiores. Dentre as variedades testadas, as variedades SP79-1011 e Java apresentaram os melhores resultados de cinética de produção de gases e nutrientes digestíveis totais comparativamente superiores.

PALAVRAS-CHAVES: Degradabilidade verdadeira in vitro, produção de gases, Saccharum officinarum.

\section{ABSTRACT}

\section{FRACTIONATION AND KINETICS OF in vitro RUMINAL FERMENTATION OF THE CARBOHYDRATES OF FIVE SUGAR- CANE VARIETIES}

The objective of this study was to determine the chemical composition, the carbohydrates fractionation and the kinetics of in vitro ruminal fermentation of fibrous and non-fibrous carbohydrates of five sugarcane varieties. Variety SP79-1011 presented the highest value of total digestible nutrients $(65.9 \%)$, followed by the varieties Java, RB72454, SP80-1842 and RB765418. For the accumulated gas in the dry matter, 48 and 96 horas of in vitro incubation were higher for the variety SP79-1011, showing significant difference in comparison with the varieties RB765418, RB72454 and SP80-
1842, excepting the variety Java that did not show any difference in relation the first one. The dry matter partitioning factor decreased with the incubation time and there was no difference among the evaluated varieties. The characteristics of the production cycle influenced the in vitro ruminal fermentation of the evaluated varieties, being the varieties of precocious cycle (SP80-1842 and RB765418) the ones with the worst results. Amongst the varieties tested, SP79-1011 and Java presented the best results of gas production kinetics and total digestible nutrients.

KEYWORDS: In vitro true degradability, gas production, Saccharum officinarum. 


\section{INTRODUÇÃO}

A partir do final do século XX, o Brasil tornouse o maior produtor mundial de cana-de-açúcar, com safra em 2007 de 516 milhões de toneladas, em área plantada de 6,7 milhões de hectares (IBGE, 2008). A sua maior utilização é para fabricação de açúcar e álcool. No entanto, a cana-de-açúcar tem atraído cada vez mais a atenção dos pecuaristas, sendo usada sazonalmente como alimento volumoso principal, pelo fato de apresentar as seguintes características: elevada produção por unidade de área cultivada, cultivo relativamente fácil, baixo custo por unidade de matéria seca (MS) produzida e coincidência do período de sua maior disponibilidade com o período de escassez de forragem na forma de pasto (LANDELL et al., 2002).

A produtividade animal é determinada basicamente pela qualidade da forragem ingerida. Ela é fortemente influenciada pela digestibilidade da forragem, a qual é inversamente proporcional ao conteúdo da fração fibrosa das plantas (carboidratos fibrosos). A baixa digestão ruminal da forragem ingerida aumenta o tempo de retenção do alimento no rúmen e, consequentemente, diminui a taxa de ingestão de matéria seca e o desempenho animal.

Os carboidratos representam a principal reserva da energia fotossintética nos seres fotoautotróficos. Estes compostos constituem cerca de $60 \%$ a $80 \%$ da matéria seca das forrageiras, sendo a principal fonte de energia para os seres vivos compreendidos nos primeiros níveis tróficos. Para os ruminantes, eles tornam-se disponíveis indiretamente, por intermédio do processo fermentativo de origem microbiana no rúmen-retículo na forma de ácidos graxos voláteis (AGV) e, diretamente, pela absorção de seus monômeros constituintes, no trato gastrintestinal desses animais (VAN SOEST, 1994).

Atualmente, novos sistemas de avaliação de alimentos para ruminantes indicam a necessidade de diferenciar as frações que compõem os carboidratos e compostos nitrogenados nos alimentos, com o intuito de possibilitar a predição do crescimento microbiano no rúmen, degradação ruminal dos alimentos e o desempenho animal (RODRIGUES \& VIEIRA, 2006),

Em termos nutricionais, os carboidratos podem ser classificados como carboidratos não fibrosos e carboidratos fibrosos. Os primeiros são completamente disponíveis no rúmen e os segundos apresentam-se parcialmente disponíveis (MERTENS, 1997).

Adicionalmente, tendo em vista as limitações de consumo da cana-de-açúcar provocadas pelas características de sua fração fibrosa (AZEVÊDO et al., 2003; FERNANDES et al., 2003), torna-se importante conhecer a qualidade de diferentes variedades, em relação aos teores de fibra e de variáveis da cinética de degradação ruminal da FDN, com o objetivo de poder selecionar as melhores variedades, confrontando-as em estudos sobre suas características químicas, cinética da fermentação ruminal e testes de desempenho animal.

A técnica in vitro semiautomática de produção de gases apresenta potencial em descrever a cinética da fermentação no rúmen, fornecer a taxa e a extensão da degradação das forrageiras, bem como medir produtos da fermentação de partes solúveis e insolúveis de substratos. Essa técnica permite avaliar grande número de substratos por experimento, apresentando alta precisão nas medições, simplicidade no manuseio de equipamentos e baixo custo na implantação e por amostra analisada (MAURÍCIO et al., 1999).

Objetivou-se com o presente trabalho, determinar a composição química, o fracionamento dos carboidratos e a cinética da fermentação ruminal in vitro das frações de carboidratos não fibrosos (CNF) e carboidratos fibrosos (CF) de cinco variedades de cana-de-açúcar.

\section{MATERIAL E MÉTODOS}

O experimento de campo foi desenvolvido na Fazenda Experimental da Escola Agrotécnica Federal de Salinas, localizada no município de Salinas, MG, situado na latitude $16^{\circ} 10^{\prime}$ sul e longitude de $42^{\circ} 18^{\prime}$ oeste a uma altitude média de $472 \mathrm{~m}$. O clima da região corresponde na classificação de Koppen ao tipo AW.

As variedades avaliadas foram Java, RB72-454, SP79-1011 (ciclo médio-tardia) e RB76-5418, SP801842 (ciclo precoce). A escolha das variedades ocorreu por já estarem sendo cultivadas por agricultores da região. Foi utilizado o delineamento em blocos completos casualizados, composto de cinco tratamentos (variedades) e quatro repetições. Cada parcela ou unidade de amostragem foi constituída de seis linhas com $10 \mathrm{~m}$ de comprimento, no espaçamento de 1,3 m entre linhas, com uma área total de $78 \mathrm{~m}^{2}$. As mudas 
utilizadas apresentavam idade entre dez e doze meses e foram picadas em toletes que continham de três ou quatro gemas, distribuindo-se manualmente em sulcos. $\mathrm{O}$ solo da área experimental foi classificado como Latossolo Vermelho-Escuro, com textura média distrófico tendo como composição química $\mathrm{pH}$ em água 5,4; $\mathrm{P}$ de $5 \mathrm{mg} / \mathrm{dm}^{3} ; \mathrm{K}$ de $0,63 \mathrm{cmol}_{\mathrm{c}} / \mathrm{dm}^{3} ; \mathrm{Al} \mathrm{de} 0,1 \mathrm{cmol}_{\mathrm{c}} /$ $\mathrm{dm}^{3} ; \mathrm{Ca}$ de 4,3 $\mathrm{cmol}_{\mathrm{c}} / \mathrm{dm}^{3} ; \mathrm{Mg}$ de $2 \mathrm{cmol}_{\mathrm{c}} / \mathrm{dm}^{3} ; \mathrm{H}+\mathrm{Al}$ de $2,9 \mathrm{cmol}_{\mathrm{c}} / \mathrm{dm}^{3}$ e V 70\%. Realizou-se o plantio no dia 13 de novembro de 2003, sendo a colheita realizada entre os meses de maio e junho de 2004, ocasião em que as variedades apresentaram um grau Brix em média de $20 \%$.

As análises químicas e os ensaios de fermentação ruminal in vitro dos colmos inteiros das cinco variedades foram realizadas no Campus da Universidade Estadual do Sudoeste da Bahia, no Laboratório de Nutrição Animal, em Vitória da Conquista, BA.

Após a colheita, realizou-se a picagem individual dos colmos inteiros das cinco variedades de canade-açúcar. Os colmos foram posteriormente levados para a estufa de circulação forçada de ar a $55^{\circ} \mathrm{C}$ por 72 horas e moídos em moinho de facas tipo "Wiley" em peneira com crivos de $1 \mathrm{~mm}$ de diâmetro. Constituiuse um pool nas amostras obtidas formando amostras simples das variedades. Estas amostras foram utilizadas nos ensaios de cinética da fermentação ruminal in vitro, pela técnica semiautomática de produção de gases (MAURÍCIO et al., 1999).

As análises químicas para a determinação dos teores percentuais de matéria seca (MS), proteína bruta (PB), extrato etéreo (EE) e matéria mineral (MM) foram realizadas seguindo procedimentos padrões da AOAC (1990). Determinaram-se as análises de fibra em detergente neutro (FDN), fibra em detergente ácido (FDA) e lignina (em ácido sulfúrico a 72\%) conforme GOERING \& VAN SOEST (1970), SILVA \& QUEIROZ (2004).

As estimativas das frações que compõem os carboidratos totais (CT) foram determinadas conforme SNIFFEN et al. (1992), calculados como segue:

$\mathrm{CT}(\%)=100-(\% \mathrm{~PB}+\% \mathrm{EE}+\% \mathrm{MM})$, em que $\mathrm{PB}$ corresponde à proteína bruta; $\mathrm{EE}$ ao extrato etéreo e MM à matéria mineral da amostra.

Os carboidratos não fibrosos (CNF) foram calculados como segue:

$\mathrm{CNF}(\%)=100-(\% \mathrm{~PB}+(\% \mathrm{FDNcp})+\% \mathrm{EE}$ $+\% \mathrm{MM})$, em que FDNcp corresponde à fração FDN corrigida para o conteúdo analisado da matéria mineral e nitrogênio multiplicado pelo fator 6,25.

A fração "C" foi obtida multiplicando-se o teor de lignina pelo fator 2,4. A fração "B2" (fibra disponível) foi obtida diminuindo-se a fibra em detergente neutro, corrigido para cinzas e proteína (FDNcp), da fração "C".

Para a realização dos ensaios de fermentação in vitro da fração dos carboidratos fibrosos (CF), procedeu-se ao preparo inicial das amostras, que consistiu na realização prévia de uma análise de fibra em detergente neutro (FDN), pesando-se três gramas de amostra e digerindo-se em $300 \mathrm{~mL}$ de solução de detergente neutro (GOERING \& VAN SOEST, 1970; SILVA \& QUEIROZ, 2004). O resíduo da filtração em cadinhos com porosidade $\mathrm{n}^{\circ} 1(50 \mathrm{~mL})$ foi, então, lavado sequencialmente cinco vezes com água quente, duas vezes com acetona e outras cinco vezes com água quente, no intuito de retirar todo o resíduo de detergente que viesse a prejudicar a fermentação in vitro.

As incubações foram realizadas separadamente para a matéria seca (MS) dos colmos inteiros e para os resíduos da FDN destes. Em frascos de $160 \mathrm{~mL}$, adicionaram-se $\mathrm{CO}_{2}, 1 \mathrm{~g}$ de amostra $(1 \mathrm{~mm})$ e $90 \mathrm{~mL}$ de meio de cultura (THEODOROU et al., 1994; MAURÍCIO et al., 1999). Os animais doadores de fluido ruminal foram mantidos com alimentação diária de $1 \mathrm{~kg}$ de farelo de trigo, capim napier (P. purpureum) e cana-de-açúcar picados (1:1 na matéria natural) $a d$ libitum.

Utilizaram-se réplicas para os colmos inteiros e os resíduos da FDN, perfazendo um total de quatro frascos por variedade e mais dois frascos contendo apenas fluido ruminal e meio de cultura (saliva artificial), que foi empregada como controle.

Os horários de leituras da produção de gases foram de 2, 4, 6, 8, 10, 12, 14, 17, 20, 24, 28, 32, 48, 72, e 96 horas, após o início do processo de fermentação in vitro. Os tempos de degradação in vitro foram de 12, 24, 48 e 96 horas, sendo os resíduos de colmos inteiros e as frações de CF das cinco variedades de cana-de-açúcar, após a fermentação in vitro, filtrados em cadinhos com porosidade $n^{\circ} 1$. Realizaram-se as leituras de pressão de forma semiautomática com a ajuda de um transdutor de pressão tipo T443A.

Com o intuito de se obter a produção de biomassa microbiana e o fator de partição, procedeu-se à 
avaliação da degradabilidade verdadeira in vitro nas diferentes amostras. Em uma repetição das amostras, determinaram-se as degradabilidades verdadeiras in vitro (MS e resíduo da FDN), digerindo-se o resíduo da fermentação em $100 \mathrm{~mL}$ de solução de FDN por uma hora, filtrando-se o resíduo em cadinho $\mathrm{n}^{\circ} 1 \mathrm{e}$ secando-o a $105^{\circ} \mathrm{C}$ por 24 horas (GOERING \& VAN SOEST, 1970). Na outra repetição, determinou-se a degradabilidade aparente in vitro da matéria seca, sendo os resíduos da fermentação filtrados diretamente em cadinhos $\mathrm{n}^{\circ} 1$, que foram então colocados em estufa a $105{ }^{\circ} \mathrm{C}$ por 24 horas (MAURÍCIO et al., 1999).

A biomassa microbiana (mg.100 $\mathrm{mg}^{-1}$ de MS digestível) foi obtida diminuindo-se a degradabilidade verdadeira in vitro da degradabilidade aparente in vitro. Calculou-se o fator de partição entre a relação do substrato verdadeiramente degradado $(\mathrm{mg})$ e o volume de gases produzidos $(\mathrm{mL})$, em cada tempo de degradação (BLUMMEL et al., 1997; BLUMMEL et al., 1999).

Para determinação dos valores de nutrientes digestíveis totais (NDT) das cultivares de cana, procedeu-se aos cálculos de acordo com WEISS (1993), em nível de mantença, como segue:

NDT $(\%)=0,98 *(100-\% \mathrm{FDNn}-\% \mathrm{~PB}-$ $\% \mathrm{MM}-\% \mathrm{EE}-(0,7 * \% \mathrm{PIDA})+((\exp (-0,0012 \times$ $\% \mathrm{PIDA})) * \% \mathrm{~PB})+2,70(\% \mathrm{EE}-1)+0,75 *((\% \mathrm{FDNn}$ - \%LIG) *(1- (\%LIG / \%FDNn $\left.\left.)^{0,667}\right)\right)-7$; em que o coeficiente de digestibilidade da proteína bruta é calculado usando-se a proteína insolúvel em detergente ácido (PIDA), por intermédio da equação DVPB (\%) $=\exp (-0,0012 * \%$ PIDA $) ; \mathrm{PB}$, a proteína bruta; EE, a extrato etéreo; FDNn, a fibra em detergente neutro corrigido para nitrogênio; MM, a matéria mineral; LIG, a lignina. Todos os valores, exceto da proteína bruta ligada à fibra em detergente neutro e ácido (\% da PB), devem ser expressos como percentagem da matéria seca.

Para o cálculo dos parâmetros da produção de gases foi utilizado o modelo bicompartimental proposto por SCHOFIELD et al. (1994), descrito a seguir:

$\mathrm{V}=\mathrm{Vf} 1 /(1+\exp (2-4 * \mathrm{C} 1 *(\mathrm{~T}-\mathrm{L})))+\mathrm{Vf} 2$ / $(1+\exp (2-4 * \mathrm{C} 2 *(\mathrm{~T}-\mathrm{L})))$, em que Vf1 equivale ao volume máximo de produção de gases dos carboidratos não fibrosos (CNF); $\mathrm{C} 1$ corresponde à taxa de degradação (\%.h-1) da mesma fração; Vf2 refere-se ao volume máximo de produção de gases da fração dos carboidratos fibrosos (CF); C2 representa a taxa de degradação (\%.h-1) dos CF; e T e L referem-se aos tempos de incubação (em horas) e à latência (em horas), respectivamente.

O delineamento experimental dos ensaios de fermentação in vitro foi em blocos casualizados, em que os tratamentos consistiram nas cinco variedades de cana-de-açúcar testadas, e as repetições, os três animais doadores de fluido ruminal. As médias foram comparadas pelo teste Tukey a $5 \%$ de probabilidade com o auxílio do programa SAS (1996).

As estimativas dos parâmetros descritos no modelo matemático foram desenvolvidas utilizando-se métodos interativos não lineares. Estes resultados ajustados, por estimativas de quadrados mínimos, foram obtidos a partir do método Gauss - Newton, dentro do procedimento NLIN, com o auxílio do programa SAS (1996).

\section{RESULTADOS E DISCUSSÃO}

Os valores de matéria seca (MS), matéria mineral (MM), proteína bruta (PB), extrato etéreo (EE), fibra em detergente neutro (FDN), fibra em detergente ácido (FDA) e lignina (LIG) variaram entre $26,8 \%$ e $30,1 \%$, $1,4 \%$ e $2,3 \%, 1,6 \%$ e $2,8 \%, 0,37 \%$ e $0,59 \%, 39,0 \%$ e $46,4 \%, 22,8 \%$ e $29,3 \%$ e $3,9 \%$ e $7,4 \%$, respectivamente (Tabela 1). Esses resultados estão dentro da faixa de variação apresentada por VALADARES FILHO et al. (2006). A maior variação ocorreu nos teores de FDN, FDA e LIG, alterando, como consequência, os teores de carboidratos não fibrosos (CNF), fibra disponível (B2), fibra indisponível (C) e nutrientes digestíveis totais (NDT).

Para a estimativa dos nutrientes digestíveis totais (NDT) a variedade SP79-1011 apresentou o maior valor $(65,9 \%)$, seguida das variedades Java, RB72454, SP80-1842 e RB765418 (64,0; 60,8; 57,3 e 54,3\%, respectivamente). Os teores de CNF influenciaram os valores de NDT das variedades avaliadas, já que esses carboidratos apresentam quase que completa disponibilidade nutricional para os ruminantes (VAN SOEST, 1994).

MELLO et al. (2006), avaliando a composição química de nove variedades de cana-de-açúcar, encontraram valores de MS, MM, PB, EE, FDN, FDA, CT, $\mathrm{CNF}, \mathrm{B} 2$ e $\mathrm{C}$ variando entre $22,6 \%$ e $26,9 \%, 2,3 \%$ e 
$3,5 \%, 1,9 \%$ e $3,3 \%, 0,61 \%$ e $0,89 \%, 44,2 \%$ e $52,1 \%$ $28,4 \%$ e $33,5 \%, 92,8 \%$ e $95,2 \%, 41,0 \%$ e $50,1 \%, 31,4 \%$ e $38,3 \%, 12,1 \%$ e $14,8 \%$, respectivamente. Esses dados são concordantes com os do presente trabalho.
A PAGMS às 48 e 96 horasoras da variedade SP79-1011 foi maior $(\mathrm{P}<0,05)$ em comparação com as variedades RB765418, RB72454 e SP80-1842 (Tabela 2).

TABELA 1. Composição química de cinco variedades de cana-de-açúcar

\begin{tabular}{lccccc}
\hline \multirow{2}{*}{ Itens (\%MS) } & \multicolumn{5}{c}{ Variedades } \\
\cline { 2 - 5 } & Java & RB72454 & RB765418 & SP79-1011 & SP80-1842 \\
\hline MS (\%) & $26,8 \pm 0,38$ & $28,5 \pm 0,65$ & $28,8 \pm 0,34$ & $29,4 \pm 0,87$ & $30,1 \pm 1,30$ \\
MM (\%) & $2,0 \pm 0,37$ & $2,1 \pm 1,22$ & $1,9 \pm 1,12$ & $2,3 \pm 0,84$ & $1,4 \pm 0,07$ \\
PB (\%) & $2,7 \pm 0,10$ & $2,8 \pm 0,01$ & $2,3 \pm 0,05$ & $1,6 \pm 0,08$ & $2,9 \pm 0,30$ \\
EE (\%) & $0,50 \pm 0,09$ & $0,37 \pm 0,10$ & $0,37 \pm 0,09$ & $0,45 \pm 0,04$ & $0,59 \pm 0,20$ \\
FDN (\%) & $40,1 \pm 0,33$ & $42,4 \pm 0,26$ & $46,4 \pm 0,72$ & $39,0 \pm 0,55$ & $45,8 \pm 0,69$ \\
FDA (\%) & $24,5 \pm 1,60$ & $26,4 \pm 0,70$ & $29,3 \pm 0,90$ & $22,8 \pm 0,43$ & $28,5 \pm 0,30$ \\
LIG (\%) & $4,5 \pm 0,03$ & $5,3 \pm 0,09$ & $7,4 \pm 0,03$ & $3,9 \pm 0,71$ & $6,4 \pm 0,18$ \\
CT (\%) & 94,8 & 94,7 & 95,7 & 95,7 & 95,1 \\
CNF (\%) & 56,2 & 54,0 & 50,6 & 57,3 & 50,7 \\
B2 (\%) & 27,8 & 28,0 & 27,3 & 29,0 & 29,0 \\
C (\%) & 10,8 & 12,7 & 17,8 & 9,4 & 15,4 \\
NDT (\%) & 64,0 & 60,8 & 54,3 & 65,7 & 57,3 \\
\hline
\end{tabular}

${ }^{1}$ NDT estimado de acordo com Weiss (1993) para 1x exigência de mantença. NDT $(\%)=0,98 *(100-\% \mathrm{FDNn}-\% \mathrm{~PB}-\% \mathrm{MM}-\% \mathrm{EE}-(0,7 * \% \mathrm{PIDA})$ $+((\exp (-0,0012 \times \%$ PIDA $)) * \% \mathrm{~PB})+2,70(\% \mathrm{EE}-1)+0,75 *\left((\% \mathrm{FDNn}-\% \mathrm{LIG}) *\left(1-(\% \mathrm{LIG} / \% \mathrm{FDNn}){ }^{0,667}\right)\right)-7$.

Não foi realizada análise estatística, dada a necessidade de se realizar um pool nas parcelas para os ensaios de fermentação in vitro.

TABELA 2. Valores médios de produção acumulada de gases (PAGMS; mL), fator de partição (FPMS; mg.mL $\mathrm{mL}^{-1}$ ), e biomassa microbiana (BIOMS; mg.100 $\mathrm{mg}^{-1}$ de MS digestível) da matéria seca (MS) de cinco variedades de cana-de-açúcar às 12, 24, 48 e 96 horas de incubação in vitro

\begin{tabular}{|c|c|c|c|c|c|c|}
\hline \multirow{2}{*}{ Itens } & \multicolumn{5}{|c|}{ Variedades } & \multirow{2}{*}{$\begin{array}{l}\text { CV } \\
(\%)\end{array}$} \\
\hline & Java & RB72454 & RB765418 & SP79-1011 & SP80-1842 & \\
\hline PAGMS12 & $104,5^{\mathrm{a}}$ & $98,3^{\mathrm{ab}}$ & $88,1^{\mathrm{c}}$ & $102,3^{\mathrm{ab}}$ & $96,3^{b}$ & 2,28 \\
\hline PAGMS24 & $147,0^{\mathrm{a}}$ & $139,4^{\mathrm{ab}}$ & $125,2^{\mathrm{c}}$ & $147,5^{\mathrm{a}}$ & $136,8^{\mathrm{b}}$ & 8,43 \\
\hline PAGMS48 & $197,7^{\mathrm{ab}}$ & $187,9^{\mathrm{bc}}$ & $167,2^{\mathrm{d}}$ & $202,1^{\mathrm{a}}$ & $182,5^{\mathrm{c}}$ & 2,12 \\
\hline PAGMS96 & $236,4^{a b}$ & $225,9^{\mathrm{bc}}$ & $201,6^{\mathrm{d}}$ & $241,2^{\mathrm{a}}$ & $220,0^{c}$ & 1,65 \\
\hline FPMS $12^{1}$ & $5,5^{\mathrm{a}}$ & $5,7^{\mathrm{a}}$ & $5,9^{\mathrm{a}}$ & $5,7^{\mathrm{a}}$ & $5,7^{\mathrm{a}}$ & 2,67 \\
\hline FPMS24 & $4,2^{\mathrm{a}}$ & $4,3^{\mathrm{a}}$ & $4,4^{\mathrm{a}}$ & $4,2^{\mathrm{a}}$ & $4,3^{\mathrm{a}}$ & 2,63 \\
\hline FPMS48 ${ }^{1}$ & $3,5^{\mathrm{a}}$ & $3,7^{\mathrm{a}}$ & $3,8^{\mathrm{a}}$ & $3,5^{\mathrm{a}}$ & $3,6^{\mathrm{a}}$ & 2,47 \\
\hline FPMS96 $^{1}$ & $3,1^{\mathrm{a}}$ & $3,2^{\mathrm{a}}$ & $3,2^{\mathrm{a}}$ & $3,2^{\mathrm{a}}$ & $3,2^{\mathrm{a}}$ & 2,13 \\
\hline BIOMS12 & $4,37^{\mathrm{a}}$ & $4,20^{\mathrm{a}}$ & $5,93^{\mathrm{a}}$ & $3,93^{\mathrm{a}}$ & $5,13^{\mathrm{a}}$ & 21,44 \\
\hline BIOMS24 & $1,80^{\mathrm{a}}$ & $3,37^{\mathrm{a}}$ & $2,47^{\mathrm{a}}$ & $3,47^{\mathrm{a}}$ & $3,70^{\mathrm{a}}$ & 66,36 \\
\hline BIOMS48 & $3,87^{\mathrm{a}}$ & $4,53^{\mathrm{a}}$ & $4,87^{\mathrm{a}}$ & $2,83^{\mathrm{a}}$ & $2,37^{\mathrm{a}}$ & 37,29 \\
\hline BIOMS96 & $2,85^{\mathrm{a}}$ & $2,27^{\mathrm{a}}$ & $2,30^{\mathrm{a}}$ & $2,33^{\mathrm{a}}$ & $2,57^{\mathrm{a}}$ & 34,04 \\
\hline
\end{tabular}

Médias seguidas por letras distintas na linha diferem entre si pelo teste Tukey a $5 \%$ de probabilidade $(\mathrm{P}<0,05)$.

${ }^{1} \mathrm{mg}$ do substrato verdadeiramente degradado/volume de gases produzidos em $\mathrm{mL}$. 
NOGUEIRA et al. (2006) avaliaram a cinética da fermentação ruminal in vitro de diferentes forrageiras pela técnica semiautomática de produção de gases e encontraram valores médios de produção acumulada de gases ao término do período de incubação in vitro para a cana-de-açúcar de $263 \mathrm{~mL}$, sendo similares aos descritos na Tabela 2.

AZEVÊDO et al. (2003) também observaram que as variedades de ciclo precoce foram inferiores, sendo as variedades de ciclo de produção médiotardia (SP79-1011 e RB845257) as que apresentaram PAGMS superior em relação às de ciclo de produção precoce (SP80-1842). Este resultado pode ser explicado pelo fato de as variedades de ciclo de produção médio-tardia poderem fornecer mais energia para os microrganismos que fermentam os carboidratos não fibrosos (CNF), apresentando-se mais eficientes na síntese de proteína microbiana.

Os valores do fator de partição da MS (FPMS), que relacionam a quantidade de substrato verdadeiramente degradado $(\mathrm{mg})$ e a produção de gases $(\mathrm{mL})$, diminuíram com o tempo de incubação entre as variedades avaliadas $(\mathrm{P}>0,05)$.

Não foi encontrada diferença significativa para os valores de BIOMS às 96 horas de incubação in vitro, que variaram de 2,27 a $2,85 \mathrm{mg} .100 \mathrm{mg}^{-1}$ de substrato verdadeiramente degradado. A PAGFDN às 12, 24, 48 e 96 horas de incubação foi maior $(\mathrm{P}<0,05)$ para a variedade RB72454 em relação às variedades SP79-1011 e RB765418 às 96 horas de incubação (Tabela 3).

A elevada variabilidade dos resultados encontrados para FPFDN e BIOFDN, refletida nos altos coeficientes de variação $(\mathrm{CV})$, dificultou a detecção de diferenças entre as variedades $(\mathrm{P}>0,05)$. Esta alta variação ocorreu provavelmente em virtude das dificuldades nas mensurações gravimétricas dos resíduos antes e após o tratamento com detergente neutro.

Os resultados apresentados neste trabalho estão de acordo aos observados por SCHOFIELD \& PELL (1995), que avaliaram (48 horas de incubação in vitro) as curvas de produção total de gases pela metodologia de subtração das curvas no capim-colonião (Panicum maximum).

NOGUEIRA et al. (2006) avaliaram a fermentação in vitro da MS e do material lavado em água da cana-de-açúcar pela técnica semiautomática de produção de gases e também encontraram um perfil de fermentação in vitro semelhante ao deste estudo, caracterizado pela fermentação mais rápida dos CNF, e mais lenta da fração fibrosa da cana-de-açúcar.

TABELA 3. Valores médios de produção acumulada de gases (PAGFDN; mL), fator de partição (FPFDN; mg.mL $\left.{ }^{-1}\right)$ e biomassa microbiana (BIOFDN; mg.100 $\mathrm{mg}^{-1}$ da MS digestível) da fibra em detergente neutro (FDN) de cinco variedades de cana-de-açúcar às 12, 24, 48 e 96 horas de incubação

\begin{tabular}{|c|c|c|c|c|c|c|}
\hline \multirow{2}{*}{ Itens } & \multicolumn{5}{|c|}{ Variedades } & \multirow{2}{*}{ CV $(\%$} \\
\hline & Java & RB72454 & RB765418 & SP79-1011 & SP80-1842 & \\
\hline PAGFDN12 & $6,9^{c}$ & $38,8^{\mathrm{a}}$ & $6,4^{\mathrm{c}}$ & $8,4^{\mathrm{c}}$ & $16,2^{\mathrm{b}}$ & 8,85 \\
\hline PAGFDN24 & $13,7^{\mathrm{c}}$ & $50,2^{\mathrm{a}}$ & $11,4^{\mathrm{c}}$ & $12,8^{\mathrm{c}}$ & $26,9^{b}$ & 8,02 \\
\hline PAGFDN48 & $34,1^{\mathrm{bc}}$ & $72,4^{\mathrm{a}}$ & $22,2^{\mathrm{c}}$ & $25,0^{\mathrm{c}}$ & $47,4^{b}$ & 13,15 \\
\hline PAGFDN96 & $59,7^{\mathrm{ab}}$ & $94,4^{\mathrm{a}}$ & $38,3^{b}$ & $47,3^{b}$ & $65,4^{\mathrm{ab}}$ & 20,75 \\
\hline FPFDN12 $^{1}$ & $3,0^{\mathrm{a}}$ & $0,3^{\mathrm{a}}$ & $4,4^{\mathrm{a}}$ & $1,8^{\mathrm{a}}$ & $1,2^{\mathrm{a}}$ & 86,41 \\
\hline FPFDN241 & $3,9^{\mathrm{a}}$ & $0,6^{\mathrm{a}}$ & $5,1^{\mathrm{a}}$ & $1,7^{\mathrm{a}}$ & $1,1^{\mathrm{a}}$ & 78,42 \\
\hline FPFDN48 $^{1}$ & $3,3^{\mathrm{a}}$ & $1,1^{\mathrm{a}}$ & $3,3^{\mathrm{a}}$ & $3,0^{\mathrm{a}}$ & $1,8^{\mathrm{a}}$ & 31,81 \\
\hline FPFDN96 $^{1}$ & $2,6^{\mathrm{a}}$ & $1,3^{\mathrm{a}}$ & $2,9^{\mathrm{a}}$ & $2,4^{\mathrm{a}}$ & $2,0^{\mathrm{a}}$ & 22,90 \\
\hline BIOFDN12 & $1,77^{\mathrm{a}}$ & $1,13^{\mathrm{a}}$ & $2,20^{\mathrm{a}}$ & $1,17^{\mathrm{a}}$ & $2,17^{\mathrm{a}}$ & 44,45 \\
\hline BIOFDN24 & $2,87^{\mathrm{a}}$ & $1,63^{\mathrm{a}}$ & $1,80^{\mathrm{a}}$ & $0,77^{\mathrm{a}}$ & $2,40^{\mathrm{a}}$ & 82,86 \\
\hline BIOFDN48 & $3,53^{\mathrm{a}}$ & $3,73^{\mathrm{a}}$ & $3,57^{\mathrm{a}}$ & $5,90^{\mathrm{a}}$ & $4,17^{\mathrm{a}}$ & 45,08 \\
\hline BIOFDN96 & $2,93^{\mathrm{a}}$ & $3,60^{\mathrm{a}}$ & $3,43^{\mathrm{a}}$ & $\mathrm{Nd}$ & $4,13^{\mathrm{a}}$ & 30,98 \\
\hline
\end{tabular}

Médias seguidas por letras distintas na linha diferem entre si pelo teste Tukey a $5 \%$ de probabilidade $(\mathrm{P}<0,05)$.

${ }^{1} \mathrm{mg}$ do substrato verdadeiramente degradado/volume de gases produzidos em $\mathrm{mL}$.

nd: não determinado. 
Entre as variedades de cana avaliadas não houve diferença significativa $(\mathrm{P}>0,05)$ em relação aos parâmetros cinéticos de produção de gases in vitro. FERNANDES et al. (2003), avaliando as taxas de digestão dos carboidratos de variedades de cana-de-açúcar com diferentes ciclos de produção (precoce e intermediário), obtiveram valores para Vf1, Vf2, $\mathrm{C} 1$ e C2 de 98,8 e $98,6 \mathrm{~mL} \cdot \mathrm{g}^{-1}, 168,6$ e $170,6 \mathrm{~mL} \cdot \mathrm{g}^{-1}, 0,182$ e $0,185 \cdot \mathrm{h}^{-1}$, 0,023 e $0,023 . h^{-1}$, respectivamente. Os valores de C1 e
$\mathrm{C} 2$ foram semelhantes aos deste estudo $(0,182$ a 0,220 .$h^{-1}$ e 0,021 a $\left.0,023 . h^{-1}\right)$, porém os valores de Vf1 e Vf2 foram ligeiramente acima dos valores apresentados neste trabalho. Para possibilitar a comparação dos parâmetros da cinética de fermentação in vitro, os resultados (Vf1 e Vf2) de FERNANDES et al. (2003) foram extrapolados para $1 \mathrm{~g}$ de amostra, uma vez que utilizaram $100 \mathrm{mg}$ de amostra para incubação.

TABELA 4. Estimativas dos parâmetros cinéticos da produção de gases in vitro da matéria seca (MS) de cinco variedades de cana-deaçúcar

\begin{tabular}{lcccccc}
\hline Itens & \multicolumn{5}{c}{ Variedades } \\
\cline { 2 - 5 } & Java & RB72454 & RB765418 & SP79-1011 & SP80-1842 \\
\hline Vf1 (mL.g $\left.{ }^{-1}\right)$ & $86,9^{\mathrm{a}}$ & $79,9^{\mathrm{a}}$ & $77,4^{\mathrm{a}}$ & $85,0^{\mathrm{a}}$ & $80,6^{\mathrm{a}}$ & 11,13 \\
C1 $\left(\mathrm{h}^{-1}\right)$ & $0,214^{\mathrm{a}}$ & $0,218^{\mathrm{a}}$ & $0,182^{\mathrm{a}}$ & $0,220^{\mathrm{a}}$ & $0,195^{\mathrm{a}}$ & 13,97 \\
L (h) & $5,68^{\mathrm{a}}$ & $5,51^{\mathrm{a}}$ & $5,69^{\mathrm{a}}$ & $6,11^{\mathrm{a}}$ & $5,42^{\mathrm{a}}$ & 16,34 \\
Vf2 (mL.g-1) & $140,7^{\mathrm{a}}$ & $136,6^{\mathrm{a}}$ & $117,0^{\mathrm{a}}$ & $147,1^{\mathrm{a}}$ & $130,9^{\mathrm{a}}$ & 12,14 \\
C2 $\left(\mathrm{h}^{-1}\right)$ & $0,022^{\mathrm{a}}$ & $0,022^{\mathrm{a}}$ & $0,021^{\mathrm{a}}$ & $0,023^{\mathrm{a}}$ & $0,022^{\mathrm{a}}$ & 5,91 \\
\hline
\end{tabular}

Médias seguidas por letras distintas na linha diferem entre si pelo teste Tukey a $5 \%$ de probabilidade $(\mathrm{P}<0,05)$.

Vf1: volume máximo de produção de gases da fração dos CNF; C1 - taxa de digestão para a fração dos CNF; L: tempo de colonização; Vf2: volume máximo de produção de gases da fração dos CF; C2: taxa de digestão para a fração dos CF.

CAMPOS et al. (2001), em avaliação da produção de gases in vitro de diferentes alimentos para ruminantes com sistema de monitoramente automático descritos por PELL \& SCHOFIELD (1993), encontraram estimativas dos parâmetros cinéticos de produção de gases para a variedade RB72454 de 9,8 mL.g $\mathrm{g}^{-1}, 7,5 \mathrm{~mL} \cdot \mathrm{g}^{-1}, 0,20 . \mathrm{h}^{-1}, 0,031 . \mathrm{h}^{-1}$ e 1,3 h para Vf1, Vf2, C1, C2 e L, respectivamente. Os parâmetros da cinética de produção de gases apresentaram valores inferiores aos do presente trabalho. Isso pode ser explicado pela diferença entre as técnicas utilizadas, em que a técnica automática executada por CAMPOS et al. (2001) forneceu estimativas dos parâmetros de forma rápida (48 horas de incubação e $100 \mathrm{mg}$ de amostra).

A variedade RB72454 apresentou uma maior PAGFDN, mas com menores valores de FPFDN em relação às demais (Tabela 3 ). Esses valores de FPFDN menores implicam uma menor degradabilidade in vitro da FDN. A grande limitação da cana-de-açúcar é a baixa digestibilidade da fração dos CF (AZEVÊDO et al., 2003; NOGUEIRA et al., 2006). Assim, materiais que apresentam CF mais digestíveis podem ser utilizados em programas de melhoramento genético da cana-de-açúcar para fins forrageiros.

Na Figura 1, estão demonstradas, graficamente, de forma conjunta, a PAGMS, PAGCNF e PAGCF nos diferentes tempos de incubação para as cinco variedades de cana-de-açúcar. A variedade SP79-1011 e Java apresentaram as maiores PAGMS, seguidas das variedades RB72454, SP80-1842 e RB765418.

A maior contribuição dos CNF na produção de gases na MS apresentada neste trabalho está de acordo com diversos trabalhos publicados na literatura (SCHOFIELD \& PELL, 1995; MALAFAIA et al., 1999; CAMPOS et al., 2001; AZEVÊDO et al., 2003; NOGUEIRA et al., 2006). 

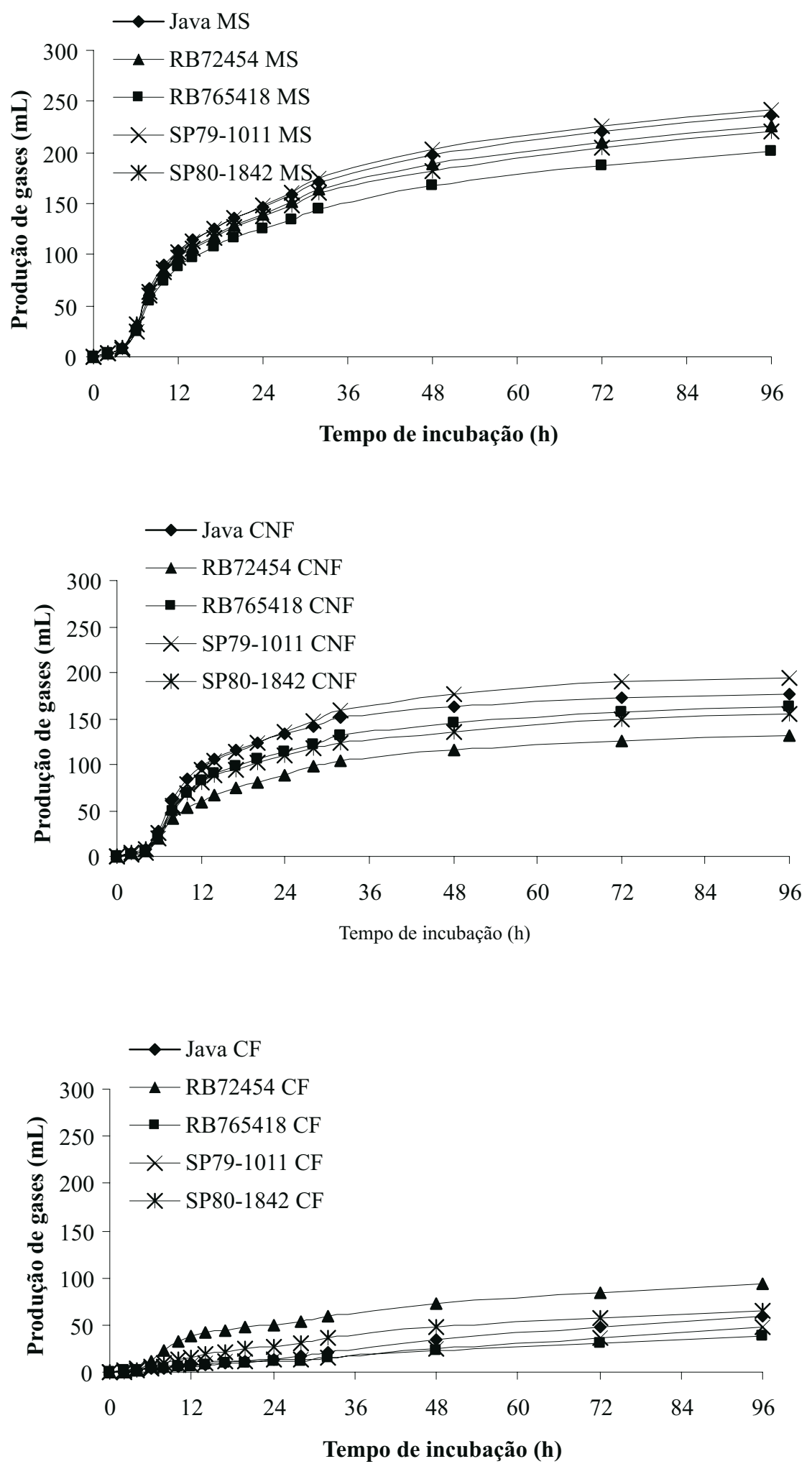

FIGURA 1. Produção acumulada de gases da matéria seca (PAGMS), dos carboidratos não fibrosos (PAGCNF) e carboidratos fibrosos (PAGCF) de cinco variedades de cana-de-açúcar. 


\section{CONCLUSÕES}

Dentre as variedades testadas, destacam-se as variedades SP79-1011 e Java, pelo fato de apresentarem melhores resultados de cinética de produção de gases e melhor composição química em relação aos CNF, C e NDT.

\section{REFERÊNCIAS}

AOAC - Association of Official Analytical Chemists. Official methods of analysis. 15. ed. Arlington, Virginia, v. 1, 1990. 1117 p.

AZEVÊDO, J. C. G.; PEREIRA, J. C.; QUEIROZ, A. C.; CARNEIRO, P. C. S.; LANA, P. L.; BARBOSA, M. H. P.; FERNANDES, A. M.; RENNÓ, F. P. Composição químico-bromatológica, fracionamento de carboidratos e cinética da degradação in vitro da fibra de três variedades de cana-de-açúcar (Saccharum spp.). Revista Brasileira de Zootecnia, v. 32, n. 6, p. 1443-1453, 2003.

BLUMMEL, M.; MGOMEZULU, R.; CHEN, X. B.; MAKKAR, H. P.; BECKER, K.; ØRSKOV, E. R. The modification of an in vitro gas production test to detect roughage related differences in vivo microbial protein synthesis as estimated by the excretion of purine derivatives. Journal of Agricultural Science, v. 133, p. 335-340, 1999.

BLUMMEL, M.; STEINGAB, H.; BECKER, K. The relationship between in vitro gas production, in vitro microbial biomass yield an ${ }^{15} \mathrm{~N}$ incorporation and its implications for the prediction of voluntary feed intake of roughages. British Journal of Nutrition, v. 77, p. 911-921, 1997.

CAMPOS, F. P.; SAMPAIO, A. A. M.; VIEIRA, P. F.; BOSE, M. L. V. Digestibilidade in vitro/ gás de volumoso exclusivos ou combinados avaliados pelo resíduo remanescente da digestão da matéria seca e produção de gás. Revista Brasileira de Zootecnia, v. 30, n. 5, p. 1579-1589, 2001.

FERNANDES, A. M.; QUEIROZ, A. C.; PEREIRA, J. C.; LANA, R. P.; BARBOSA, M. H. P.; FONSECA, D. M.; DETMANN, E.; CABRAL, L. S.; PEREIRA, E. S.; VITTORI, A. Fracionamento e cinética da degradação in vitro dos carboidratos constituintes da cana-de-açúcar com diferentes ciclos de produção em três idades de corte. Revista Brasileira de Zootecnia, v. 32, n. 6, p. 17781785, 2003. (Supl. 1)

GOERING, H. K.; VAN SOEST, P. J. Forage fiber analysis (Apparatus, reagents, procedures, and some applications). Agricultural Handbook, n. 379. Washington DC: ARS USDA, 1970.

IBGE. Levantamento sistemático da produção agrícola. Disponível em: http:/www.ibge.gov.br/ibge/estatistica/indicadores/ agropecuaria/lspa/default.sht Acesso em: 4 jul. 2008.
LANDELL, M. G. A.; CAMPANA, M. P.; RODRIGUES, A. A.; CRUZ, G. M.; ROSSETO, R.; FIGUEIREDO, P. A variedade IAC86-2480 como nova opção de cana-de-açúcar para fins forrageiros: manejo de produção de uso na alimentação animal. Boletim Técnico IAC, Campinas: Instituto Agronômico, 2002. 193 p.

MALAFAIA, P. A. M.; VALADARES FILHO, S. C.; VIEIRA, R. A. M. Kinetic parameters of ruminal degradation estimated with a non-automated system to measure gas production. Livestock Production Science, v. 58, n. 1, p. 65-73, 1999.

MAURÍCIO, R. M.; MOULD, F. L.; DHANOA, M. S. A semiautomated in vitro gas production technique for ruminant feedstuff evaluation. Animal Feed Science and Technology, v. 79, p. 321-330, 1999.

MELLO, S. Q. S.; FRANÇA, A. F. S.; LIMA, M. L. M.; RIBEIRO, D. S.; MIYAGI, E. S.; REIS, J. G. Parâmetros do valor nutritivo de nove variedades de cana-de-açúcar cultivadas sob irrigação. Ciência Animal Brasileira, v. 7, n. 4, p. 373-380, 2006.

MERTENS, D. R. Creating a system for meeting the fiber requirements of dairy cows. Journal of Dairy Science, v. 8, p. 1463-1469, 1997.

NOGUEIRA, U. T.; MAURÍCIO, R. M.; GONÇALVES, L. C. Comparação de substratos com diferentes quantidades de carboidratos solúveis utilizando a técnica in vitro semi-automática de produção de gases. Arquivo Brasileiro de Medicina Veterinária e Zootecnia, v. 58, n. 4, p. 633-641, 2006.

PELL, A. N.; SCHOFIELD, P. Computerized monitoring of gas production to measure forage digestion in vitro. Journal of Dairy Science, v. 72, p. 1063-1073, 1993.

RODRIGUES, M. T.; VIEIRA, R. A. M. Metodologias aplicadas ao fracionamento de alimentos. In: RODRIGUES, M. T.; BERCHIELLI, T. T.; PIRES, A. V.; OLIVEIRA, S. G. Nutrição de ruminantes. Jaboticabal: Funep, 2006. 583 p.

SAS. Statistical Analysis System. Software, version 6.0. Cary: SAS Institute, 1996.

SCHOFIELD, P.; PELL, A. N. Measurement and kinetic analysis of the neutral detergent-soluble carbohydrate fraction of legumes and grasse. Journal of Animal Science, v. 73, p. 3455-3463, 1995.

SCHOFIELD, P.; PITT, R. E.; PELL, A. N. Kinetics of fiber digestion from in vitro gas production. Journal of Animal Science, v. 72, n. 11, p. 2980-2991, 1994.

SILVA, D. J.; QUEIROZ, A. C. Análise de alimentos: métodos químicos e biológicos. 3. ed. Viçosa: UFV, 2004. 235 p.

SNIFFEN, C. J.; O`CONNOR, J. D.; VAN SOEST, P. J.; FOX, D. G.; RUSSELL, J. B. A net carbohydrate an protein systm for 
evaluating cattle diets: II. Carbohydrate and protein availability.

Journal of Animal Science, v. 70, p. 3562-3577, 1992.

THEODOROU, M. K.; WILLIAMS, B. A.; DHANOA, M. S.; MCALLAN, A. B. A simple gas production method using a pressure transducer to determine fermentation kinetics of ruminant feeds. Animal Feed Science Technology, v. 48, p. 185-197, 1994.

VALADARES FILHO, S. C; MAGALHÃES, K. A.; ROCHA JÚNIOR, V. R.; CAPPELLE, E. R. Tabelas brasileiras de composição de alimentos para bovinos. Viçosa: UFV, 2006. $329 \mathrm{p}$.

VAN SOEST, P. J. Nutritional ecology of the ruminant. 2 ed. Ithaca: Cornell University Press, 1994. 476 p.

WEISS, W. P. Predicting energy values of feeds. Journal of Dairy Science, v. 76, p. 1802-1811, 1993.

Protocolado em: 18 set. 2008. Aceito em: 24 ago. 2010. 